\title{
A Wireless System for Monitoring of Children with Suspected Cardiac Arrhythmias
}

\author{
Efthyvoulos Kyriacou \\ Frederick University Cyprus \\ 18 Mariou Agathaggelou Str. \\ Lemesos 3080,Cyprus \\ e.kyriacou@frederick.ac.cy \\ Marios Pattichis \\ University of New Mexico \\ Albuquerque, NM, USA \\ pattichis@eece.unm.edu \\ Kounoudes Anastasis \\ Signal Generix Ltd \\ Lemesos, Cyprus \\ tasos@signalgenerix.com
}

\author{
Constantinos Pattichis \\ University of Cyprus \\ Nicosia, Cyprus \\ pattichi@ucy.ac.cy \\ Loukas Paraskeva \\ University of Cyprus \\ Nicosia, Cyprus \\ Loucas.Paraskeva@gmail.com \\ Antonis Jossif \\ "Paedi" Center for Specialised \\ Pediatrics \\ Nicosia, Cyprus \\ ajossif@paedi.org.cy \\ Dimitris Hoplaros \\ University of Cyprus \\ Nicosia, Cyprus \\ dhoplaros@gmail.com \\ Dimitris Vogiatzis \\ University of Cyprus \\ Nicosia, Cyprus \\ dimitrv@iit.demokritos.gr
}

\begin{abstract}
Arrhythmia is one of the most difficult problems in Cardiology and especially in Pediatric Cardiology. In this study, we present a mobile health (m-Health) system that will be used for continuous monitoring of children with suspected cardiac arrhythmias. The system is able to do real-time acquisition and transmission of ECG signals, and facilitate an alarm scheme able to identify possible arrhythmias so as to notify the on-call doctor and the relatives of the child that an event or something that denotes malfunction is happening.
\end{abstract}

In general the problem has been divided into two cases. The first one, called "In-house case" the subject is located in his/her house. While for the second, called "Moving patient case" the subject might be located anywhere else. Our goal is the continuous 24 hours monitoring of the child. During the "In house case", a sensor network installed in the child's house will be used in order to continuously monitor ECG signals from the patient as well as environmental parameters. The second case is more general. For this case, the child will be monitored using the same ECG recording device but the signals will be transmitted, through a PDA device, directly to the central monitoring system. The transmission is performed through the use of $2.5 \mathrm{G}$ and $3 \mathrm{G}$ mobile communication networks.

The system design, development and technical tests (using simulator) are finished. The next step will be the better verification of the system on healthy volunteers so as to get ready for application on patients.

Permission to make digital or hard copies of all or part of this work for personal or classroom use is granted without fee provided that copies are not made or distributed for profit or commercial advantage and that copies bear this notice and the full citation on the first page. To copy otherwise, or republish, to post on servers or to redistribute to lists, requires prior specific permission and/or a fee.

PETRA'09, June 09-13, 2009, Corfu, Greece.

Copyright 2009 ACM ISBN 978-1-60558-409-6...\$5.00.

\section{Categories and Subject Descriptors}

J. 3[Computer Applications]: Life and Medical Sciences Health, Medical Information Systems.

\section{General Terms}

Management, Measurement, Design, Verification

\section{Keywords}

mobile health, sensor networks, wireless telemedicine, home monitoring, children arrhythmias.

\section{INTRODUCTION}

Telemedicine has been used for many years in order to improve health care provision or for patient monitoring solutions. Several issues such as the computational capability, size of the devices, power efficiency and cost have been limiting the availability of devices and services to a few special cases [1], [2]. Recent advancements in communications and computer systems can help us develop general-purpose systems that are more efficient, much smaller and at lower costs.

In this study, we will focus on the continuous monitoring of children with suspected cardiac arrhythmias. In order to evaluate the size and severity of the problem; arrhythmia is one of the most difficult problems in Cardiology both in terms of diagnosis and management. The problem is particularly pronounced in Pediatric Cardiology because of the variety of etiologies and the difficulty that the children are having in trying to communicate their symptoms. For example in the case of hypertrophic cardiomyopathy, it is known that children are at higher risk for arrhythmias and sudden death than adults. In most of the cases an ECG tracing is required and this is sufficient for an accurate diagnosis, whereas in some cases, a more sophisticated modality is required [1], [3]. 


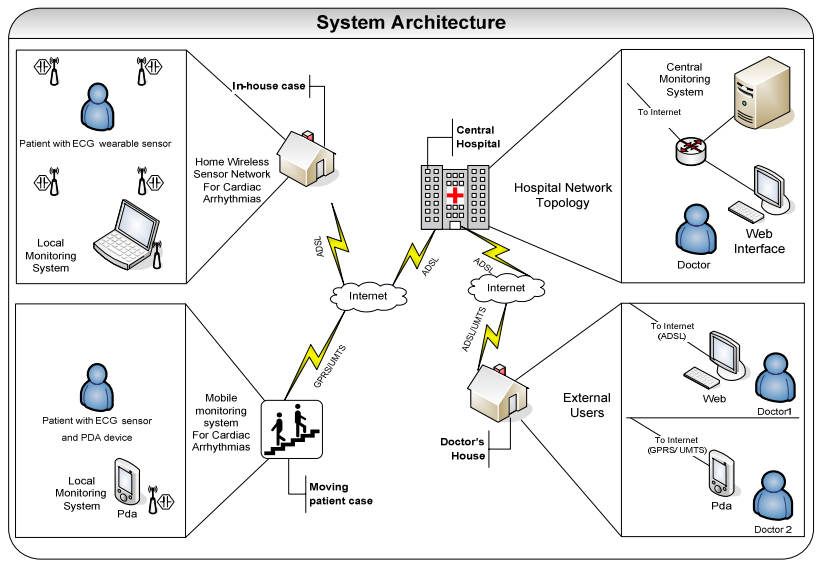

Figure 1. Overall system Architecture (C) IEEE 2007 [6]

As an example a relatively recently recognized rare form of cariomyopathy, the Isolated Noncompaction of the Left Ventricle (NCLV), which is a rare form of cardiomyopathy, poses new challenges. A subset of patients with this disease are especially prone to arrhythmia and sudden death. It is not always possible to estimate the risk of each patient with the available test modalities even if we include genetic testing. Current testing with the holter monitor has proved insufficient because it is limited to 24 or 48 hours of recording during which the patient may be asymptomatic. We care for a group of such children, some of whom are at imminent risk of sudden death [1], [4].

In this study, a mobile health (m-Health) system that will be able to monitor children continuously during their daily life activities is proposed. The system will be able to do real-time acquisition and transmission of ECG signals from the patient, and facilitate an alarm scheme able to identify possible arrhythmias so as to notify the on-call doctor and the relatives of the child that an event or something that denotes malfunction is happening. This system is a significant extension over our earlier telemedicine work in real-time ambulatory monitoring systems [5].

\section{EXAMPLE CASES}

In order to better appreciate the problem a brief description of two cases is presented:

\subsection{Case 1}

A few years ago we lost one such child, a four-year-old boy that was our first patient to be diagnosed with this disease. He was presented with a near miss episode of sudden death while at the nursery school and he was revived at the hospital where he was brought unconscious. He was then referred to UK for electrophysiological studies that were essentially normal. He had periods of bradycardia on the holter monitor and during his hospitalization. It was believed that the episode of loss of consciousness may have been related to bradycardia and a pacemaker was implanted. A few months later, while at school, he lost consciousness again and was brought to the hospital but he could not be revived this time. Before dying, an ominous form of arrhythmia (torsades de pointe) was recorded. A few weeks before death, his mother reported a very short episode of near loss of

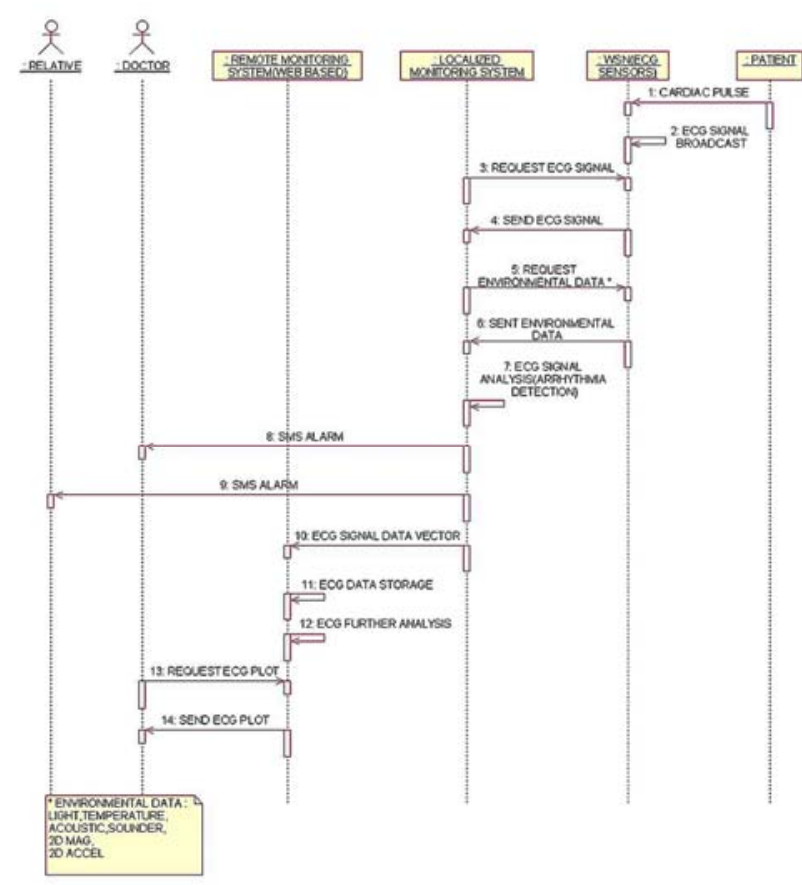

Figure 2. UML - sequence diagram of the actions being performed during the in-house case $\odot$ IEEE 2007 [6]

consciousness that retrospectively could have been a short episode of arrhythmia but unfortunately there was no record of it.

\subsection{Case 2}

The second case is that of a nine year old girl with a history of several episodes of loss of consciousness and NCLV. In her case, we were lucky to record one such episode on the holter monitor. She had multiple episodes of supravenrticular tachycardia following one of which there was a prolonged pause that

coincided with the witnessed episode of loss of consciousness. She was then started on beta blockers and she remains asymptomatic over the last three years.

\subsection{Motivation}

The description of these two cases highlights the wide range of clinical presentation and the variety of the underlying arrhythmias that these children present. Some of these children are high risk for sudden death and at the same time it is very difficult to decide for the proper treatment, making their ECG monitoring a very important task. The methods available today for ECG monitoring are clearly insufficient for this purpose. We need a noninvasive or minimally invasive way to record the ECG for extended periods of time and at the same time perform automatic analysis continuously or at frequent intervals.

\section{METHODOLOGY}

In general the problem has been divided into two cases. The first one, called "In-house case" the subject is located in his/her house. While for the second, called "Moving patient case" the subject might be located anywhere else. Our goal is the continuous 24 hours monitoring of the child. 


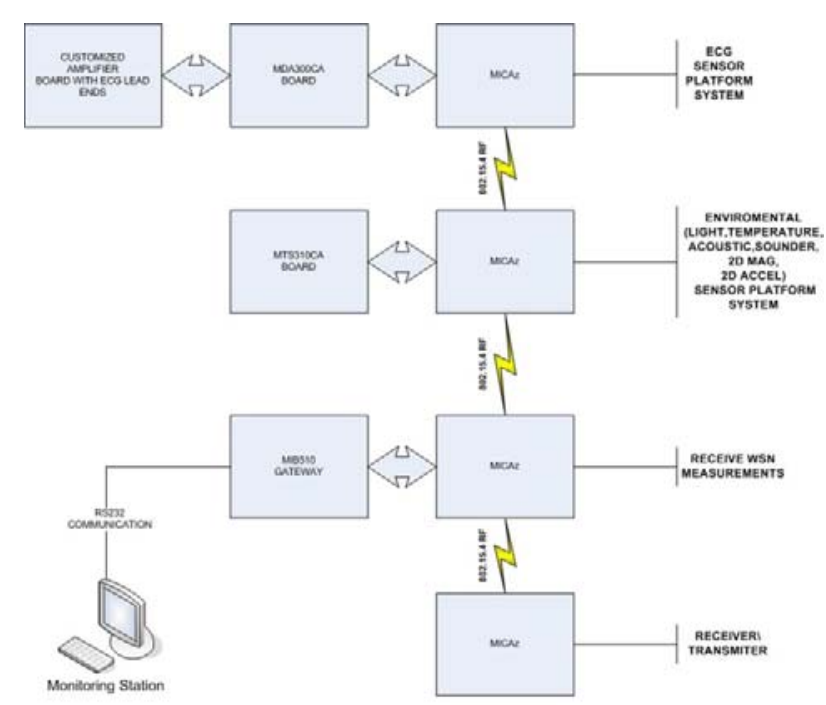

Figure 3. Block diagram of the in-house wireless sensor network (c) IEEE 2007 [6]

An overall architecture diagram of the proposed system can be seen in figure 1 . On the left hand side the two cases of patient monitoring are displayed; while on the right hand site the doctors and the access to the system are displayed.

\subsection{In-house case}

During this case, a sensor network installed in the child's house that will be used in order to continuously monitor ECG signals

from the patient [6] - [10]. Several other environmental parameters like light, temperature, sound, acceleration will also be monitored so as to continuously check the living conditions. The ECG (3 lead) signal will be recorded by a sensor carried from the child, that is part of a wireless sensor network (WSN) installed in the house. The ECG sensor has been specially designed \& developed by SignalGenerix ltd (http://www.signalgenerix.com) Signal information from the wearable sensor is propagated to a local monitoring station which will also act as a gateway to the rest of the monitoring network. This case can be described as follows (see Figure 2):

The cardiac pulse is propagated through the WSN to the local monitoring station with an embedded broadcast algorithm.

The local monitoring station is responsible for collecting environmental measurements (e.g. temperature, 2D accelerometer, sound, light):

- Sample the ECG signal.

- Store the sensor data locally.

- Analyze the ECG signal in order to detect possible cardiac arrhythmias.

In the case of a detected arrhythmia:

- Send an alarm message to the central monitoring station (located in hospital).

- Send an alarm via an SMS to the supervising doctor and a relative.

The central monitoring station is responsible to:

- Store data sent from the local monitoring station.

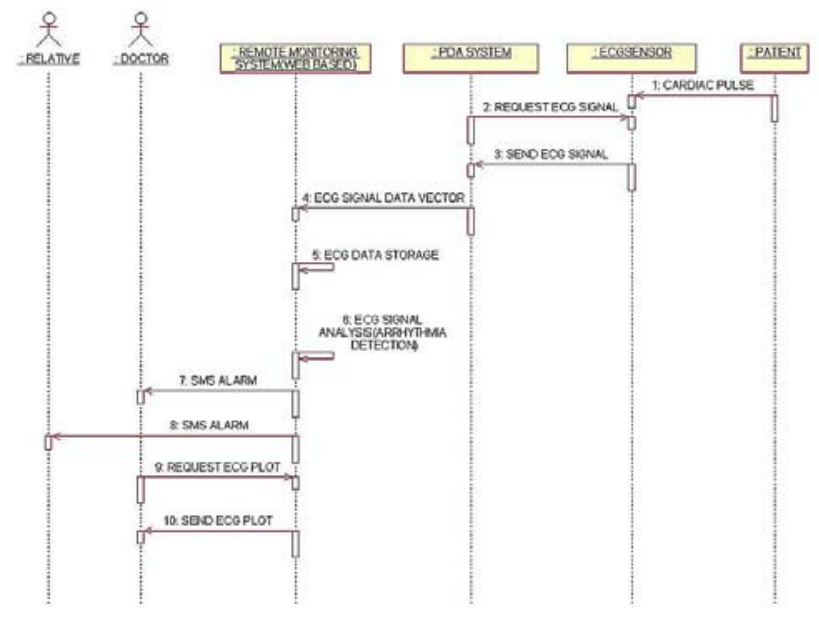

Figure 4. UML - sequence diagram of the actions performed for the Moving patient case $\subset$ IEEE 2007 [6]

- Display data transmitted from the local monitoring stations and through a web interface.

- Analyze the ECG signal further. (send a message (SMS, email etc.) to the doctor)

For our case we have chosen to develop a Mote-based sensor network based on Crossbow ${ }^{\circledR}$ equipment [9], [11]. The proposed network that will be used to cover the patient's house is based on motes like MicaZTM while the acquisition of ECG data is performed through a custom created board connected to the MDA300CA ${ }^{\mathrm{TM}}$ acquisition board. Additional environmental data will be collected through the MTS310CA ${ }^{\mathrm{TM}}$ sensor board. All collected information will be transmitted to a gateway, MIB510 ${ }^{\mathrm{TM}}$ connected on a Personal Computer; this is going to be the local monitoring system (see Figure 3).

\subsection{Moving patient case}

The second case is more general and will be used in order to complete the coverage of the system. For this case, the child will be monitored using the same ECG recording device but the

signals are transmitted, through a PDA, directly to the central monitoring system (for the test pilot case an HP iPAQ hw6915 was used). The transmission is be performed through the use of 2.5G and 3G mobile communication networks (GPRS/UMTS) [1]; depending from the equipment and network (see Figure 1).

The information is stored locally on the PDA and a basic analysis of the signals is performed locally on the mobile unit depending on the equipment specification. The central station is responsible for the storage, analysis and display of information, as well as the notification of the on-call doctor and the relative in case of an alarm. The case can be described as (see Figure 4):

The cardiac pulse is propagated through the ECG sensors to a PDA carried by the patient.

The PDA system is responsible to:

- Sample the ECG signal and send it via GPRS/UMTS to the remote monitoring station.

The remote monitoring system is responsible to: 

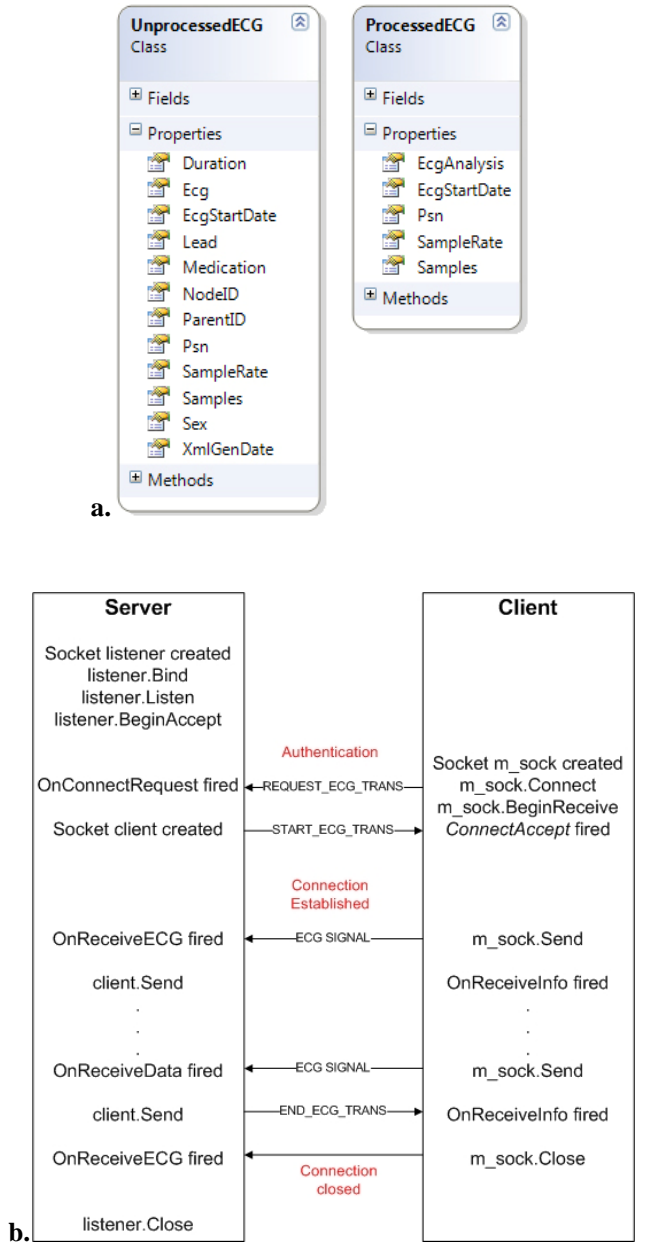

Figure 5. a. The two classes of the XML communication Scheme Unprocessed-Processed Classes. b. Transmission protocol followed during the connection of the sites to server

- $\quad$ Store data for the specific patient.

- Analyze the ECG signal in order to detect possible cardiac arrhythmias.

- Send an alarm via an SMS or e-mail to the supervising doctor and a relative.

- Display data locally and through the web.

\subsection{Variable rate ECG signal recording}

The continuous-time monitoring is limited by the available battery power. In order to achieve better results and more time of transmission we propose the use of a variable-rate signal processing system that will be used to reduce the power requirements by reducing the sampling rate during normal operation, while saving the high sampling-rate and transmission

during a possible arrhythmia session. To recognize the power savings, we note that power consumption is directly proportional to the frequency of operation. Thus, we can reduce power consumption by increasing the sampling period $T_{s}$. We can show that without changing the anti-aliasing analog filter prior to sampling, using a digital filter of variable bandwidth $B W$, we

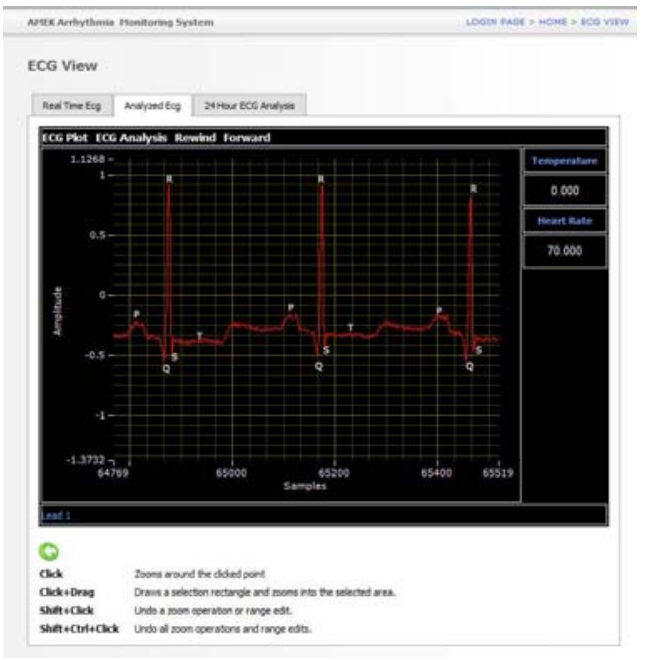

Figure 6. Snapshot from a sample transmitted ECG signal with the analysis points

can produce a properly sampled ECG signal, sampled at 2(1-1/ BW $) T_{S}$. Furthermore, larger sampling periods can be accommodated by varying the analog cutoff frequency of the antialiasing analog, lowpass filter. Here, we note that we do require continuous transmission of one lead of ECG signal. For the case of a moving patient, three leads will be acquired and stored on the device, but only one waveform will be transmitted continuously [8]. This will result in power savings due to the reduction in transmission power requirements.

\subsection{ECG Analysis and Transmission}

For the ECG analysis needs of our project we use the open source QRS Detection software provided by E.P.Limited online at [12]. The software uses two modules. The first one is the QRS Detection which includes the QRS detection functions and the QRS filter functions. The second is the beat classification module which is used for beat classification and detection.

For the ECG signal transmission between the in-house network and the remote monitoring system an asynchronous $\mathrm{xml}$ communication protocol is proposed. The protocol is based on two important classes shown in Figure 5.a. The first is the Unprocessed ECG class which is responsible to collect all the data related to the recorded ECG. The processed ECG class is responsible to collect all the data related to the analyzed ECG.

The Unprocessed ECG class contains the following properties: Psn is patient's identification number, ECG corresponds to a set of values for the ECG signal ex $(200,150,175 \ldots)$, EcgStartDate is the timestamp of the first ECG sample value in Ecg field, SampleRate for our system is $200 \mathrm{~Hz}$, Samples is the number of ECG samples, XmlGenDate is the timestamp when the xml file is generated, Sex, NodeID and ParentID contain information about the sensor network, Duration is the ECG signal duration, Lead and Medication. Processed ECG class is consisted from the Psn EcgStartDate, SampleRate, Samples and EcgAnalyis properties. In EcgAnalysis field the analysis information (ex P,P,P,Q,R,S) is stored.

Based on the above classes a client in home monitoring and a server in remote motoring system are built so that after the ECG 
Table 1. Initial evaluation tests using the ECG node and the gateway

\begin{tabular}{|c|c|c|c|c|c|}
\hline & 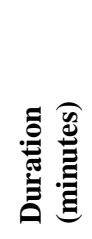 & 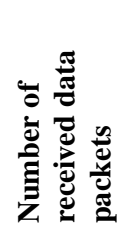 & 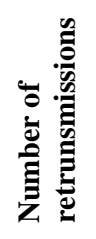 & 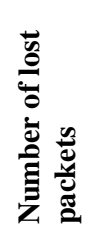 & 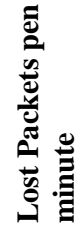 \\
\hline Test 1 & 30 & 18127 & 1049 & 17 & 0.56 \\
\hline Test 2 & 30 & 17540 & 1587 & 62 & 2.06 \\
\hline Test 3 & 30 & 16269 & 5263 & 348 & 11.6 \\
\hline Test 4 & 40 & 20548 & 42792 & 3553 & 88.83 \\
\hline
\end{tabular}

signal is recorder and or analyzed to be transmitted to the remote server. The protocol is based on the TCP/IP protocol suite and asynchronous communications. The sequence of the messages that are exchanged in the cases of Unprocessed ECG and Processed ECG signal is shown in Figure 3.b. First the client transmits a request message to start sending ECG signal. The server receives the message and transmits a message to the client in order to start the ECG signal transmission. The connection is closed when the client receives a message to stop drop the connection.

An example of the transmitted signal after the online analysis is show in Figure 6.

\section{RESULTS}

In order to verify the correct transmission of data over the system evaluate the system and verify several tests have been performed. The first set of tests was for the in-house use of the system. Tests were performed using ECG simulator with a sensor node connected to the gateway.

Four different types of tests were initially performed and the mean values can be seen on table 1 .

The types of tests performed are:

Test 1: The transmitting node is not moving. It is in a distance of three meters from the gateway, in the same room.

Test 2: The transmitting node is not moving. It is in a distance of five meters from the gateway, in the same room.

Test 3: The transmitting node is not moving. It is in a distance of six meters from the gateway, in a next door room. Room doors are open.

Test 4: The transmitting node is not moving. It is in a distance of six meters from the gateway, in a next door room. Room doors are close.

During these initial tests we have observed that the further we moved from the gateway the more retransmission we had. This is the expected behavior of the system. The encouraging fact was that despite the retransmission the quality of the signal was ok and only some minor errors were introduced.

Furthermore some connection tests of the in-house network to the main server were performed. These were performed by connecting a house to the central server using ADSL lines (server had $1 \mathrm{Mb} / \mathrm{s}$ down-stream and $128 \mathrm{kbps}$ up-stream, and house had
$2 \mathrm{Mb} / \mathrm{s}$ down-stream and $512 \mathrm{~Kb} / \mathrm{s}$ up-stream). Through the tests we had transmitted packets of 1050 bytes (these included 100 blocks of ECG and environmental samples). The tests as expected had correct transmission rate up to $99 \%$.

Currently the system s going through exhausting tests related to all the fields of transmission (Sensor networks, GPRS/2G), operation constraints, such as power consumption and functional response, like arrhythmia detection.

\section{CONCLUSIONS}

Concluding, a prototype m-Health monitoring system for children with possible arrhythmias has been created. As we have discussed, identification of children with arrhythmias is not an easy task and the treatment is not the same in all cases. Some forms of treatment, as is the case for some antiarrhythmia medications, increase the risk of arrhythmia (proarrhythmia effect) so it would be wrong to start such treatment without prior documentation of the problem. Another form of treatment uses implantable defibrillators. This form of treatment has given some promising results over the last few years. As we noted, it is difficult to decide who needs to be treated, not only because of the enormous cost but also because it entails a major at risk for arrhythmia and to record the type of arrhythmia that is needed before deciding on the proper management.

We hope that through the use of such a system we will be able to help in the identification of the type of problem thus helping the doctors provide the proper treatment. We have provided the architecture description and some initial tests of the. In future we intent to finish the technical testing of the system, by doing larger scale testing and move on to basic testing of the system on healthy volunteers.

\section{ACKNOWLEDGMENTS}

This work was supported in part by the Research Promotion Foundation of Cyprus under the Grant IPE/PLHRO/0506 "Wireless Microsensors for the Detection of Cardiac Arrythmias (AMEK)"

\section{REFERENCES}

[1] Pattichis, C.S. et. al. 2002. Wireless Telemedicine Systems: An Overview. IEEE Antennas \& Propagation Magazine 44, 2 (2002) 143-153.

[2] Nugent, C. et al. 2006. ECG TELECARE: Past Present and Future. M-Health: Emerging Mobile Health Systems, Ed. By R. Istepanian, S. Laxminarayan, C.S.Pattichis. Springer (2006), 375-388.

[3] Moreira, F.C. et.al. 2006. Noncompaction of the left ventricle: a new cardiomyopathy is presented to the clinician. Sao Paulo Med. J. 124,1,(2006), 31-35.

[4] Stollberger, C., Finsterer, J., 2004. Left ventricular hypertrabeculation/noncompaction. J. Am. Soc. Echocardiogr 17,1, (2004) 91-100.

[5] Kyriacou, E. et.al. 2003. Multi-purpose HealthCare Telemedicine Systems with mobile communication link support. BioMedical Engineering OnLine., 2, 7, (2003), DOI= http://www.biomedicalengineering-online.com.

[6] Kyriacou, E., Pattichis, C.S. et.al. 2007. An m-Helath Monitoring System for Children with Suspected Arrhythmias. $29^{\text {th }}$ IEEE EMBS Conf. (Lyon, France, Sept. 2007) 1794-1797.

[7] Fensli, R. et.al. 2005. A wearable ECG-recording System for Continous Arrhythmia Monitoring in a Wireless Tele-Home-Care Situation. $18^{\text {th }}$ IEEE Sym. On Comp.Based Med. Sys. 
[8] Shnayder, V. et.al. 2005. Sensor Networks for Medical Care. Harvard University Technical Report TR-08-05

[9] Proulux, J. et.al. 2006. Development and Evaluation of a Bluetooth EKG Monitoring Sensor. $19^{\text {th }}$ IEEE Sypm. On Comp.-Based Medical sys (CBMS06).

[10] Bobbie, P. O. et.al. 2006. Telemedicine: A Mote-Based Data Acqusition System for Real Time Health Monitoring. Telehealth 2006, (Alberta, Canada).
[11] Crossbow Technology: Wireless Sensor Networks DOI= http://www.xbow.com.

[12] Open Source Arrhythmia detection software DOI= http://www.eplimited.com/ 